\title{
Integrating micro- and macroevolution of development through the study of horned beetles
}

\author{
AP Moczek \\ Department of Biology, Indiana University, 915 E. Third Street, Myers Hall 150, Bloominton, IN 47405-7107, USA
}

\begin{abstract}
A major challenge in evolutionary developmental biology is to understand how developmental evolution on the level of populations and closely related species relates to macroevolutionary transitions and the origin of evolutionary novelty. Here, I review the genetic, developmental, endocrine, and ecological basis of beetle horns, a morphological novelty that exhibits remarkable diversity both below and above the species level. Integrating from a variety of approaches three major insights emerge: the origin of beetle horns relied at least in part on the redeployment of already existing genetic, developmental and endocrine mechanisms. At the same time little to no phylogenetic distance appeared to have been necessary for
\end{abstract}

the evolution of diverse modifier mechanisms that permit substantial modulation of trait expression at different time points during development in different species, sexes, alternative male morphs or even different tissue regions of the same individual. Lastly, at least a subset of these modifier mechanisms can evolve rapidly in geographically isolated populations, apparently driven by relatively simple, and probably ubiquitous, changes in ecological conditions. I discuss the implications of these results for our understanding of the genesis of morphological novelty and diversity.

Heredity (2006) 97, 168-178. doi:10.1038/sj.hdy.6800871; published online 12 July 2006

Keywords: allometry; Distal-less; EGFR; evolutionary novelty; phenotypic plasticity; Onthophagus

\section{Beetle horns: novel and diverse}

Horns occur in species belonging to at least six families of beetles, and are most diverse in the family Scarabaeidae (Arrow, 1951). Even though common in certain beetle families, beetle horns lack obvious homology to other structures in and outside the insects (Moczek and Nagy, 2005). Beetle horns can therefore be looked at as an example of an evolutionary novelty, and we can investigate the genetic, developmental, and ecological mechanisms that have contributed to the origin of these structures. At the same time beetle horns are extraordinarily diverse, ranging from small knobs or protrusions on a beetle's head or thorax to gigantic outgrowths that account for up to $30 \%$ of a beetle's body mass and completely transform the shape of whoever bears them (Figure 1a; Mizunuma, 1999). Interestingly, dramatic diversity in size and location of horn expression is not confined to the comparison of distant families or genera, but commonly exists among different species in the same genus, such as the genus Onthophagus, and often enough within individual species, and sometimes even within a single sex (Figure 1b; Arrow, 1951; Matthews, 1972). It is this remarkable diversity within a very narrow taxonomic framework that allows us to not only investigate the genetic, developmental, and ecological mechanisms that made the origin of horns possible, but also how modifications of these mechanisms and the interactions between them, have mediated the diversification of horns below and above the species level.

Correspondence: AP Moczek, Department of Biology, Indiana University, 915 E. Third Street, Myers Hall 150, Bloomington, IN 47405-7107, USA. E-mail: armin@indiana.edu

Received 7 October 2005; accepted 5 June 2006; published online 12 July 2006

\section{Growth and development of beetle horns}

Growth and development of beetle horns have so far only been studied in detail in the dung beetle genus Onthophagus, although the main findings are likely to hold true for horned beetles in general (summarized in Figure 2). The actual growth of horns is a very dynamic process confined to an approximately 48-h time window immediately prior to the transition from larva to pupa (Emlen and Nijhout, 1999; Moczek and Nagy, 2005). During this stage, also referred to as the prepupa, larvae appear passive and static, yet internally are undergoing dramatic remodeling. $\mathrm{Up}$ to this point a layer of epidermal cells has tightly lined the entire larval cuticle, which was in fact secreted by these same epidermal cells during the most recent larval molt. As the animal nears the prepupal stage it purges its gut and the muscles inside the head and thorax undergo programmed cell death (Figure 2a). Early prepupae are then characterized by a detachment, or apolysis, of the epidermis from the cuticle, which creates a fluid filled but otherwise empty space between epidermis and cuticle. Beetle horns originate at this stage as selected epidermal regions undergo rapid cell proliferation, resulting in an often dramatic folding of new tissue into this empty space underneath the larval cuticle. Just prior to the molt to the actual pupa the epidermis begins to secrete the future pupal cuticle. During the actual molting process, or eclosion, the animal then sheds its old larval cuticle, the epidermis is free to telescope out and form the future pupal horn, and the pupal cuticle hardens within hours of eclosion. All species investigated thus far share this period of prepupal horn growth. It is also at this stage that differences in prepupal horn growth generate many of the differences in horn expression we see in adults 

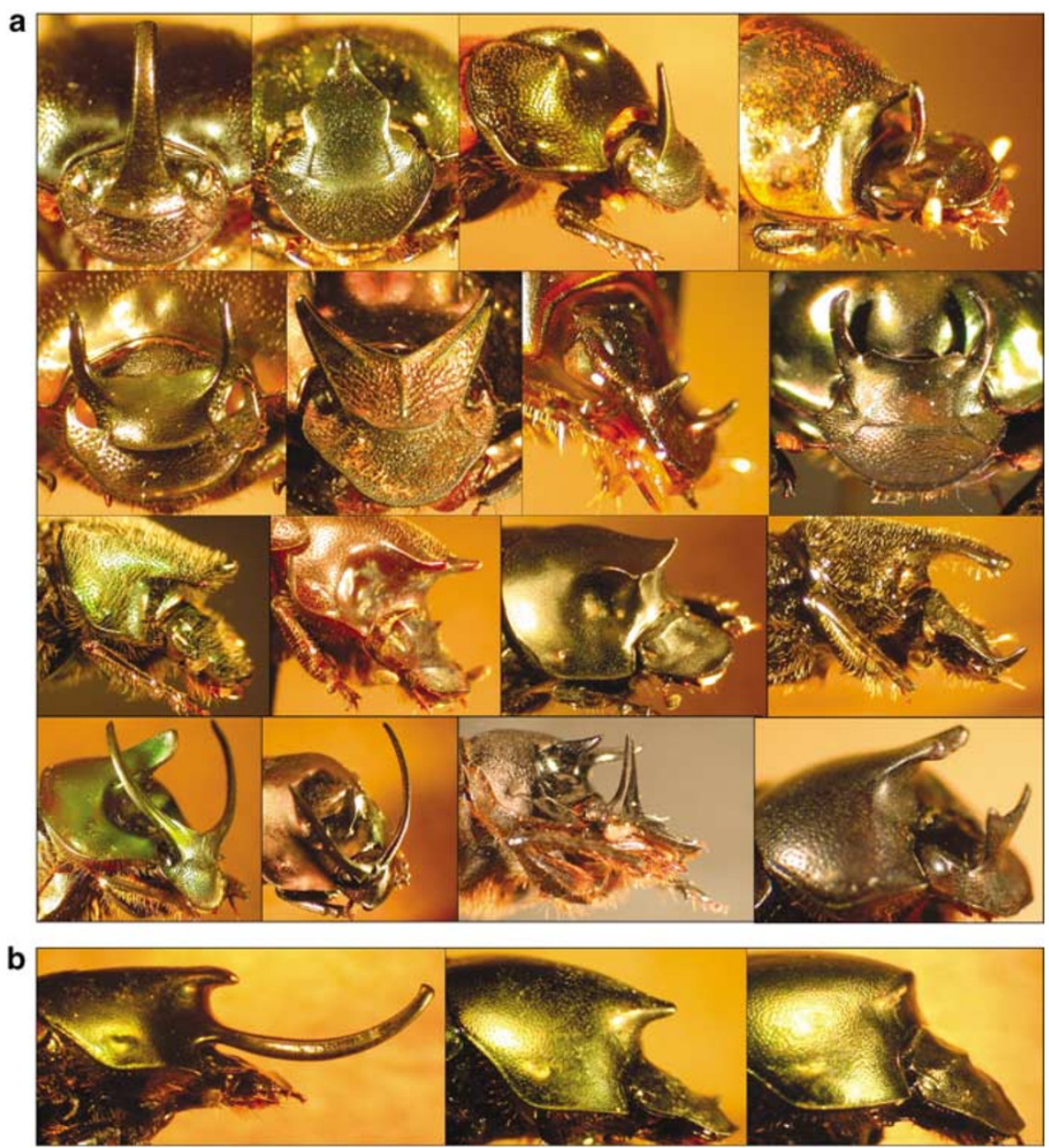

Figure 1 (a) Examples of interspecific diversity in shape, number, and location of horn expression in the genus Onthophagus. (b) One example of intraspecific diversity in horn expression in O. nigriventris (left $=$ major male, center $=$ minor male, right $=$ female; photographs: AP Moczek).

(Figure 2b). For example, in O. taurus only large adult males express a pair of head horns whereas adult females lack these horns completely. These differences in adult horn expression are already apparent in pupae, and thus due to differences in initial horn growth during the prepupal stage (Figure 3a; Moczek, in press). However, this is not true for all species, nor is it true for all horn types (Moczek and Nagy, 2005).

A second developmental period critical to defining the final size and shape of adult horns occurs during the pupal stage. In Onthophagus this stage lasts from 1 week to several weeks depending on species. During this stage, the animal in principal undergoes the same basic developmental steps as during the previous molt such as apolysis of the epidermis, secretion of a new cuticle, and eclosion to the next, now adult, stage. The main differences with respect to horn development are twofold: first, there is no major epidermal proliferation stage. Horns, just like other body parts do not grow significantly during the pupal stage. Secondly, in at least one horn type, those extending from the thorax, there is frequent differential loss of presumptive horn tissue, most likely through programmed cell death (Figure 2c and d). It is this secondary loss of horn tissue grown a few days earlier that generates sexual dimorphisms in many species, such as O. nigriventris (Moczek, in press). With the exception of the very largest males, both males and females initially grow a similar-sized horn during the prepupal stage. However, only males retain their horn into the adult stage, whereas females lose most of theirs during the pupal stage and molt into a thorax horn-less adult (Figure 3b). Curiously, in some species such as $O$. taurus (introduced above with respect to male head horns), this mechanism operates not just in one sex but in both. In this species males and females both grow a thoracic horn during the prepupal stage yet completely resorb this primordium during the pupal stage and molt into entirely thorax horn-less adult (Figure 3a). This of course raises the question of why so many species actually grow thoracic horn precursors if all they do with them is resorb them prior to the adult molt? Transient thoracic horns may reflect pleiotropic constraints or developmental left-overs of horns that ancestrally were expressed in adult. Alternatively, pupal thoracic horns may actually have a use separate from simply being an intermediary to adult horns, causing them to be maintained even in individuals that do not carry them over to the adult (Moczek, Cruikshank, Shelby, in review). All three hypotheses form the basis of current research and are likely to provide important insights 

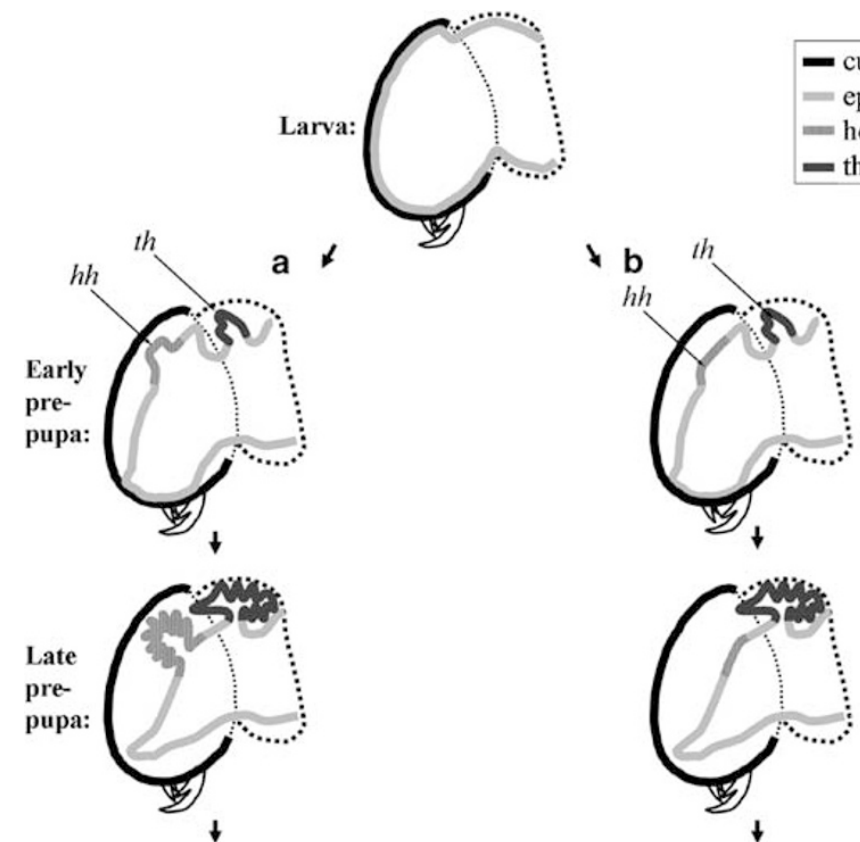

- cuticle

- epidermis

- head horn region $(\mathrm{hh})$

- thoracic horn region (th)

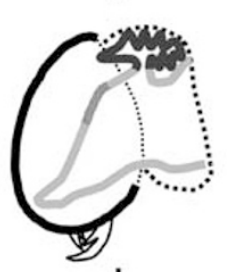

$\downarrow$
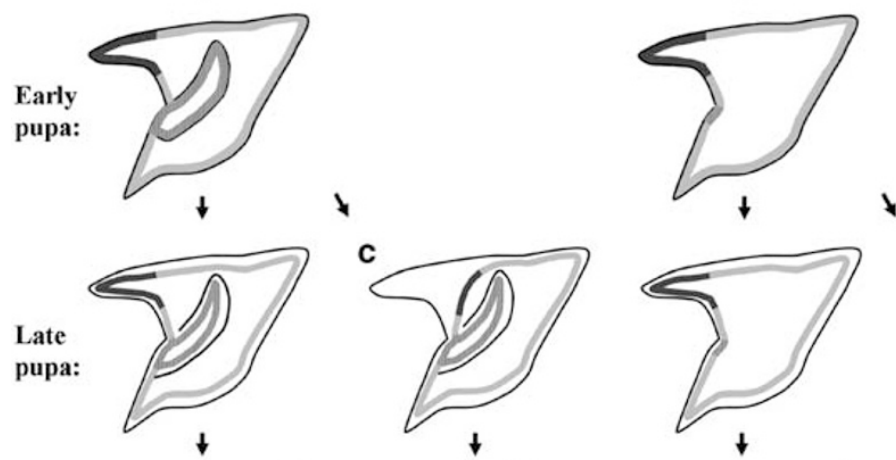

c
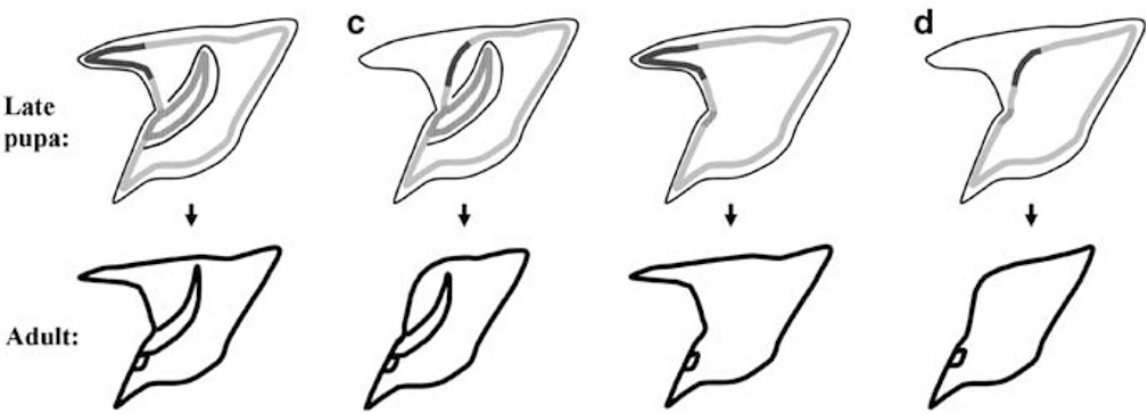

Figure 2 Development of (a) horns and (b-d) horn dimorphisms in Onthophagus beetles. (a) During the last larval instar the larval epidermis (light gray) fully lines the larval cuticle (black). At the onset of the prepupal stage the larval epidermis detaches from the cuticle (apolysis) and selected regions (shown here for a head horn (hh) and thoracic horn (th)) undergo rapid cell proliferation. The resulting extra tissue folds up underneath the larval cuticle. The epidermis subsequently secretes the future pupal cuticle, which upon the molt to the pupal stage forms the outermost layer of the pupa, lined once again by a layer of epidermal cells. During this pupal molt horn primordia are able to expand and unfold, and are now visible externally. During the second half of the pupal stage epidermal cells apolyse once more. This time, however, no significant growth of horn tissue follows apolysis. Instead, epidermal cells secrete one last cuticle and the pupa undergoes one last molt to the final adul stage. (b) Development of horn dimorphisms through differential proliferation of prepupal horn tissue (illustrated here for head horns (hh) only). During the prepupal stage presumptive horn tissue proliferates little or not at all, resulting in the absence of external horns in pupae and the resulting adults. This mechanism is used to generate sexual dimorphisms as well as alternative male morphologies for head horns in many species. (c and d) Development of horn dimorphisms through differential loss of pupal horn tissue (illustrated here for thoracic horns (th) only). Pupal horn epidermis is resorbed prior to the secretion of the final adult cuticle, most likely via programmed cell death. In many cases resorbtion of pupal horn tissue can completely erase the former presence of a thoracic horn. This mechanism contributes to sexual dimorphisms for thoracic horns in many species, and can occur in the presence or absence of (differential) head horn development.

into the evolutionary and developmental ancestry of these traits.

In summary, two discrete processes shape intra- and interspecific differences in horn expression in horned beetles: differential growth of horn tissue during the prepupal stage, and differential loss of horn primordia during the subsequent pupal stage. Interestingly, even closely related species differ dramatically in the degree to which they rely on one or the other mechanism in the genesis of intraspecific diversity.

\section{Developmental genetic regulation of beetle horns}

As outlined above, beetle horns are of epidermal origin and grow explosively during the prepupal stage. Horn development is thus at least, in part, similar to the way many insects grow their more traditional appendages, such as legs, mouthparts, or genitalia (Figure 4a and b; Fristrom and Fristrom, 1993; Nagy and Williams, 2001). 
a
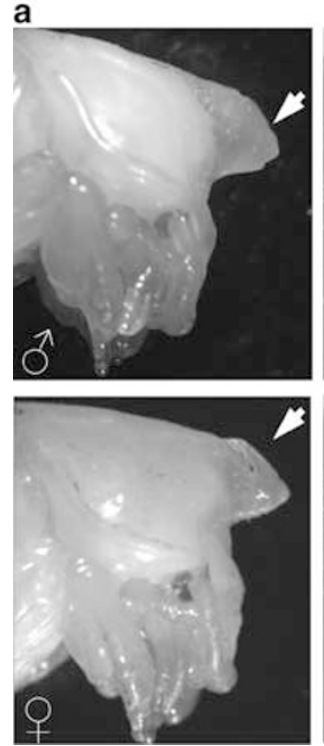
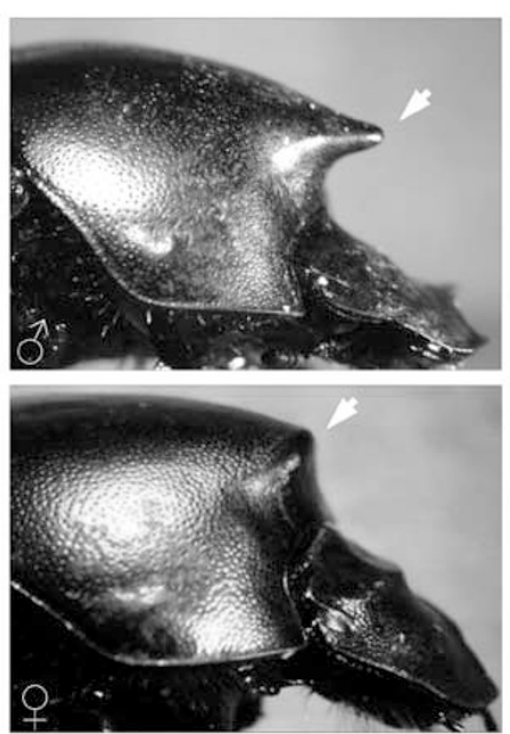

b
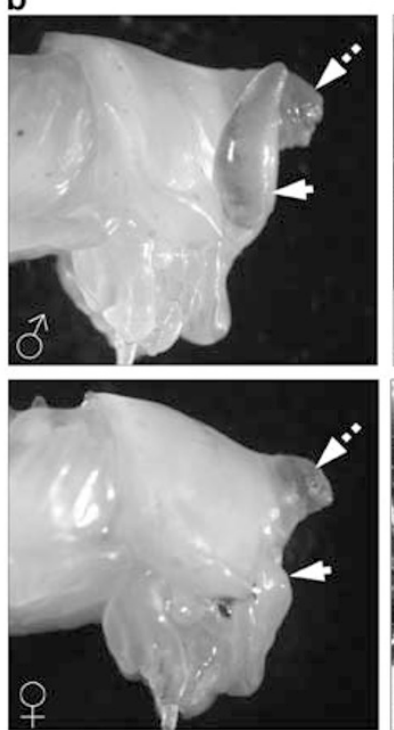
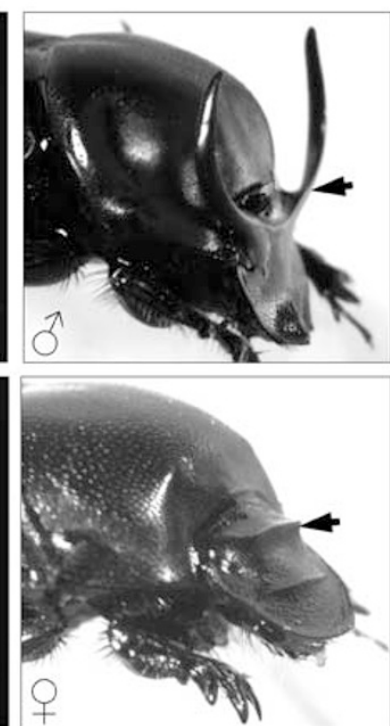

Figure 3 Sexual horn dimorphism has multiple developmental and evolutionary origins. (a) Females could have expressed horns ancestrally, but then lost them secondarily. If correct, developmental remnants of female horn expression might still be detectable. Female O. nigriventris express a transient thoracic horn as pupae (bottom, left) similar in size and shape to their male counterparts (top, left; highlighted by solid arrow) but re-absorb most of it prior to the molt to the adult (shown to the right of corresponding pupa). Females also share similar patterns of regulatory gene expression (not shown; Moczek and Nagy, 2005). These findings suggest that female hornlessness in this species may have involved the secondary sex-specific loss of horns from an originally horn-monomorphic ancestor. (b) Alternatively, the absence of horns in females could be a reflection of the ancestral character state, that is, horns evolved right away as a male-specific trait. Sexual dimorphism in head horns in species such as O. taurus may have originated in this fashion. Female O. taurus (bottom right) show no signs of horn inductive developmental events (such as pupal head horn growth or expression of patterning genes) in the same tissue regions that give rise to paired head horns in their male counterparts (highlighted by solid arrow). Striped arrows: Note that male and female O. taurus also grow a large thoracic horns clearly visible in pupae. Similar to female O. nigriventris described above, female and male O. taurus, however, resorb their entire thoracic horn during the pupal stage and molt into adults lacking any indication of the past existence of a thoracic horn (after: Moczek and Nagy (2005), Evolution E Development 7:3, 175-185).

In most insects each of these appendages originate from epidermal outbuddings roughly similar to those that give rise to horns, with the only qualitative deviation being found in the appendages of higher flies such as Drosophila and the wings of butterflies and at least some beetles (Nijhout, 1991; Tomoyasu et al, 2005). Here, appendages originate from imaginal discs, cell clusters specified and set aside during embryonic rather than late larval development. Apart from these exceptions, all other insects grow their legs, mouthparts, antennae, and genitalia the way horned beetles grow their horns (Nagy and Williams, 2001). More importantly, even though traditional insect appendages are highly diverse with respect to final morphology, location and function, and may develop from larval epidermal outbuddings or embryonic imaginal discs, they all share at least parts of a conserved network of patterning genes required for correct differentiation (reviewed in Panganiban et al (1994, 1997); Nagy and Williams (2001); Kojima (2004); Emlen and Allen (2004)). For example, formation of the proximo/distal $(\mathrm{p} / \mathrm{d})$ axis of many insect appendages relies on the subdivision of appendage primordia into separate domains of expression of the transcription factors Distall-less (Dll), dachshund (dac), homothorax (hth), and extradenticle (exd) (Figure 4). Cells expressing $D l l$ will eventually give rise to distal appendage regions, whereas dac expression typically patterns medial appendage identity, and exd/hth expression specifies proximal appendage regions that anchor appendages into the body wall. This mechanism appears conserved across a wide range of insects and noninsect arthropods and has been documented both through extensive comparative as well as functional studies (Nagy and Williams, 2001; Emlen and Allen, 2004; Kojima, 2004). This suggested the possibility that formation of the beetle horn $\mathrm{p} / \mathrm{d}$ axis may also be patterned by some of the same genes involved during the development of traditional insect appendages. This hypothesis is now strongly supported by recent developmental genetic work, which documented the expression of all four basic $\mathrm{p} / \mathrm{d}$ patterning genes, $D l l$, dac, $h t h$, and exd, during the development of prepupal horn primordia (Figure 4; Moczek and Nagy, 2005; Moczek et al, in press). Furthermore, the pattern of expression of two of these genes, Dll and $h t h$, was consistent with a conservation of patterning function, that is, Dll expression indeed occured in those regions of prepupal horn primordia that gave rise to the distal tip of adult horns while $h$ th expression was limited exclusively to proximal horn regions (Figure 4; Moczek and Nagy, 2005; Moczek et al, in press).

A second tier of genes crucial to the formation of at least some insect appendages is involved in the epidermal growth factor receptors (EGFR) signaling pathway. In Drosophila, EGFR expression forms steep gradients from the future apical tip to more proximal regions of the developing appendage (Barolo and Posakony, 2002; Campbell, 2002; Galindo et al, 2002) and graded EGFR expression regulates a suite of 
additional transcription factors such as aristaless (al), BarH1/BarH2 (bar), bric-a-brac (bab) and rotund (rn) (Campbell, 2002; Kojima, 2004). While the role of EGFR has been little studied outside Drosophila, their targets have been investigated in other arthropods. In Drosophila, aristaless patterns tarsal segment formation in legs (Campbell, 2002) and the formation of the arista on the antenna (Schneitz et al, 1993), and its ortholog in crickets is expressed in the distal portions of developing legs, mouthparts, and antennae and cerci (Miyawaki et al,
2002). Interestingly, al expression is also observed during the development of some horns, but not others. Expression of al is strong and occurs over a surprisingly large domain only in thoracic horn primordia, but is entirely absent during the development of head horns (Moczek and Nagy, 2005). An ongoing study is observing a similar pattern for other EGFR pathway genes (Kijimoto, Andrews and Moczek, in preparation). While this implicates a second traditional appendage patterning pathway in the formation of beetle horns, it also a

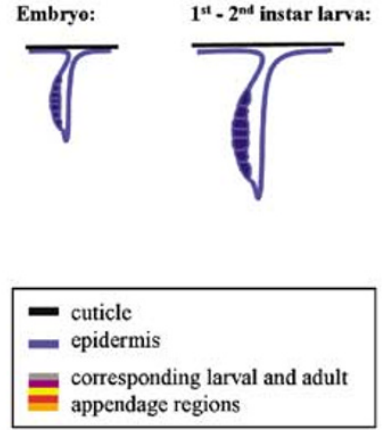

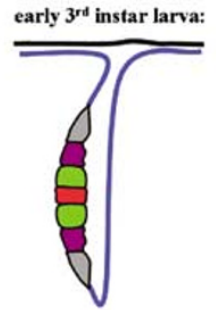
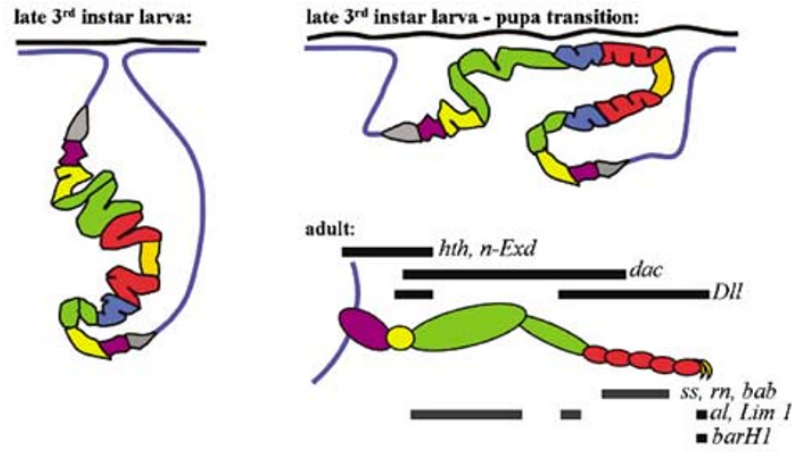

b

Embryo: $\quad 1^{\text {th }}-3^{\text {rd }}$ instar larva

late $3^{\text {rd }}$ instar (early prepupa):

late $3^{\text {rd }}$ instar (late prepupa):

pupa:
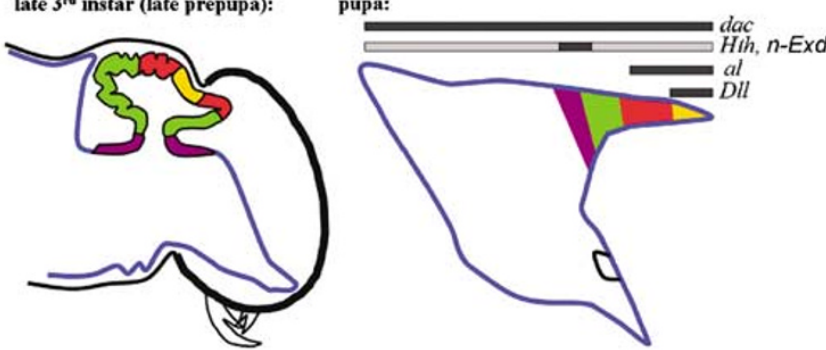

C

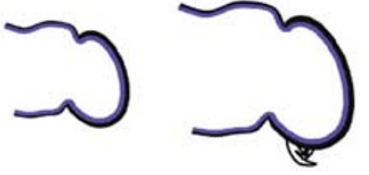

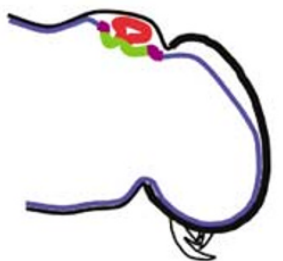

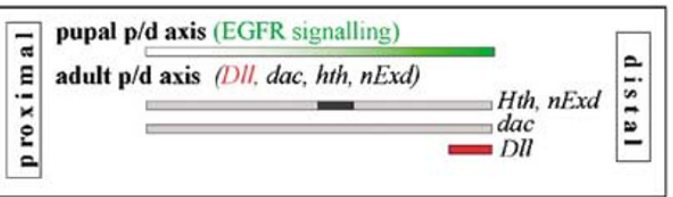

prepupa:

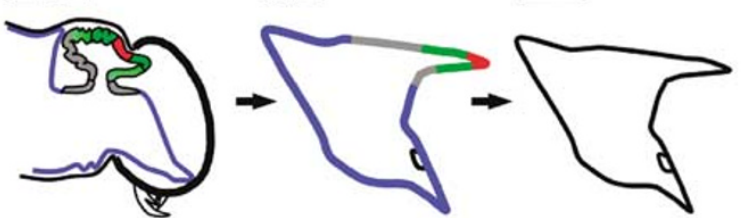

d

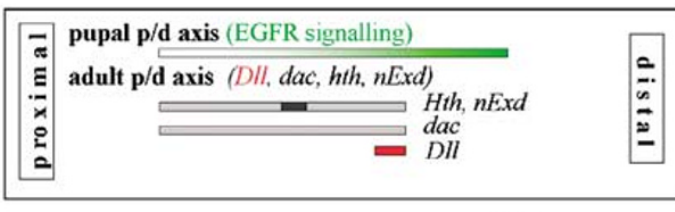

prepupa:

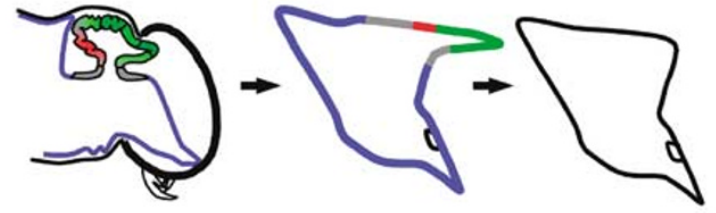

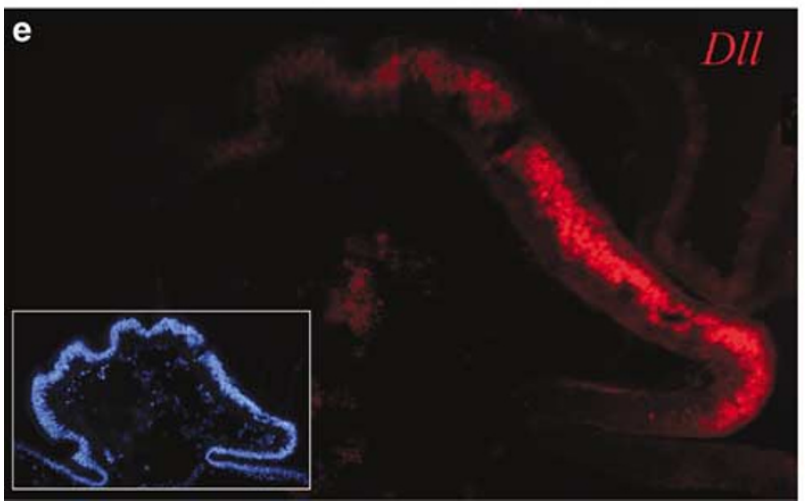

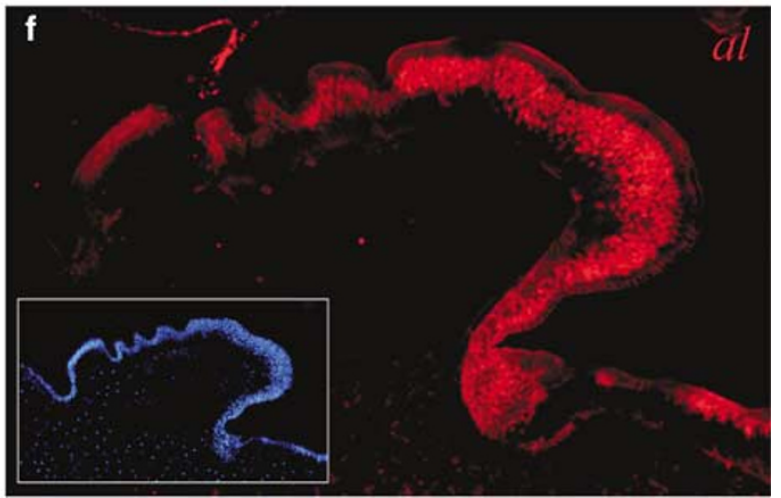


represents the first piece of developmental genetic evidence that suggests that different horn types may be patterned by different patterning genes. Different horn types may thus have different developmental origins, and may have had different and partly independent evolutionary histories (Moczek and Nagy, 2005).

Combined, these results suggest important similarities in the patterning mechanisms underlying the development of beetle horns and more tranditional insect appendages. However, recent results also suggest that certain aspects of beetle horn patterning might be of particular importance to this unique type of outgrowth, and might have played an important role in the evolution of certain aspects of intra- and interspecific diversity. For example, the exact location of DLL expression during prepupal horn development covaries tightly with differences in the degree of pupal horn resorption observed a few days later during early pupal development (Moczek and Nagy, 2005; Moczek et al, in press). The more posterior the domain of $\mathrm{Dll}$ expression during prepupal development, the greater the proportion of the pupal horn that is resorbed during pupal development. This relationship has been observed both within and between species. For example, female $O$. nigriventris exhibit far greater pupal horn resorption than their male counterparts, and also express a more posterior domain of $\mathrm{Dll}$ expression compared to males. On the other hand, in O. taurus thoracic horn resorption is severe in both sexes, which share the same posterior pattern of Dll expression. These observation suggest that the exact location of prepupal Dll expression determines the degree of pupal horn resorption via designating prepupal epidermis anterior to this proximal Dll domain for retraction. If correct, evolutionary changes in the exact location of $\mathrm{Dll}$ expression, rather than simple presence or absence of expression, would provide an important avenue for the modulation of horn expression in at least some species. This also suggests that in species such as
O. taurus, or the females of O. nigriventris (Figure 3), the $\mathrm{p} / \mathrm{d}$ axis of the pupal and adult horn are not exactly identical, but that instead the distal-most protion of the pupal horn far exceeds that of the adult (Figure $4 \mathrm{c}$ and d). If this is correct, it may suggest that during prepupal development separate, and at least partly independent patterning mechanisms may be specifying the proximodistal axis of future pupal and adult horns (Figure 4c and d). While this is clearly in need of further study, preliminary evidence in support of this notion comes from an examination of possible EGFR signaling in horn differentiation, in particular the EGFR target aristaless (al) mentioned above. al is expressed strongly in prepupal thoracic horns of O. taurus and O. nigriventris (Moczek and Nagy, 2005). Unlike Dll, however, the domain of al expression always corresponded to the distal most region of the future pupal horn, and did not differ between prepupae of the two species, or sexes within both species, regardless of the degree to which their horn primordia were subsequently resorbed during the pupal stage (Moczek and Nagy, 2005). In sum, results available to date suggest that the origin of beetle horns relied in part on the redeployment of several traditional and conserved appendage patterning elements. The same results also indicate that certain aspects of horn patterning such as the exact position and domain size of DLL expression are surprisingly evolutionarily labile and may have provided important avenues for morphological diversification below and above the species level.

\section{The origins of alternative male morphs and sexual dimorphisms}

Comparing developmental modes and patterns of gene expression across horn types present in different species is, however, only one of many informative comparisons that can provide important and often surprising insights into the origins of diversity in these organisms. One of

Figure 4 Patterning of horn development relies at least in part on the same patterning elements as the formation of more typical insect appendages such as the Drosophila leg. From left to right: Imaginal discs originate as ecdodermal invaginations and are specified during embryonic development. Patterning of the P/D axis begins in the embryo and becomes elaborated throughout larval development. In later larval development, and especially during the last instar, the leg disc undergoes dramatic growth forcing the resulting extra tissue into folds. This growth occurs while the disc remains invaginated. During the larval to pupal transition the disc evaginates, and upon pupation telescopes out to form the pupal and future adult leg. Colors indicate tissue types and regional relationships between leg disc and adult leg. Also indicated is the approximate relationship between expression domains of common P/D patterning genes during the late third instar of Drosophila and the corresponding adult leg segments (modified after Koijma, 2004, Figure 2b). (b) Schematic growth and differentiation of a thoracic horn in Onthophagus beetles. From left to right: Thus far no evidence is available that would support an embryonic or early larval phase of horn growth and patterning. Horn differentiation and growth appear confined to late larval development when animals enter the prepupal stage. At this stage the prepupal epidermis apolyses from the cuticle and horn primordia originate via explosive growth of selected epidermal tissue. This growth occurs exclusively as an evagination into the space between epidermis and cuticle freed up during the preceding apolysis. Upon pupation the thoracic horn telescopes out to form the pupal and future adult horn. Colors indicate tissue types and regional relationships between the prepupal horn primordium and the resulting pupal horn. Also indicated is the approximate relationship between the expression domains of five $\mathrm{P} / \mathrm{D}$ patterning genes thus far investigated during the prepupal stage of horn formation and the corresponding regions of the pupal prothorax. Beetle horn development therefore differs in at least two important aspects from that of imaginal disc-derived appendages as it lacks an embryonic specification and patterning phase and an invaginated larval growth phase. (c and d) Hypothetical model for the development of intra- and interspecific variation in thoracic horn expression via differential remodeling of pupal horn length based on expression data available to date. This model proposes that two separate yet temporally co-occurring patterning mechanisms specify the P/D axis of pupal and adult horns, and that both axes are at least in part developmentally and evolutionarily dissociable. In particular, this model proposes that EGFR signalling (green) patterns the $\mathrm{P} / \mathrm{D}$ axis of pupal horns irrespective of the degree to which pupal horns are retained or resorbed prior to the adult molt. In contrast, DLL (red), HTH, n-EXD, and DAC (gray) are proposed to pattern the p/d axis of the future adult prothorax irrespective of the exact pupal morphology (for HTH and n-EXD light and dark gray reflect weak and strong levels of expression, respectively). If the distal component of both axes co-localize as indicated in (c), the pupal horn morphology will match that of the adult and most of the pupal horn will be retained into the adult stage. If DLL expression occurs more posteriorly, as indicated in (d), and does not colocalize with the distal portion specified by EGFR signalling, the pupal horn morphology will not match that of the eclosing adult. In this case horn tissue regions anterior to the DLL domain become designated for resorption during pupal development, and only a small portion of the pupal horn is retained into the adult stage. (e, f): Examples of two patterning genes, (e) Distal-less and (f) aristaless, expressed during the development of the thoracic horn in O. nigriventris. Inserts show corresponding DAPI counter staining (modified after Moczek and Nagy, 2005). 
the most striking features of many horned beetle species is the extreme variation of horn expression among conspecific males. Commonly, only males with access to optimal larval feeding conditions will eclose to adults that exceed a threshold size and express fully developed horns. Smaller males, which experienced suboptimal larval feeding conditions, instead eclose to female-like, largely hornless adults. As a consequence of the threshold-size dependent expression of horns males of many species typically occur as two relatively discrete horned or hornless morphs. As size and shape of adult beetles do not change with age, these differences between males persist through their entire adult lifetime (Moczek and Emlen, 1999). Two hypotheses have been proposed regarding the origin of male horn dimorphism (Moczek, 2005). Male horn dimorphism could have originated from an ancestor in which all males were hornless, and genotypes subsequently acquired the ability to induce horn expression in males that exceeded a certain size threshold. If correct, horn-inductive developmental events such as tissue proliferation and the expression of patterning genes should then be confined to large males only. Alternatively, male horn dimorphism could have originated from an ancestor in which all males grew horns regardless of size and large males were simply enlarged versions of smaller males. Genotypes subsequently acquired the ability to inhibit horn development in small males. If correct, some horn induction might occur in all males regardless of size, but may be incomplete or repressed in small males only. Thus far the answer is clear cut and the same across species and horn types: small males show signs of horn growth and patterning similar to their large, fully horned counterparts, suggesting that facultative, size-dependent male horn dimorphisms are indeed a secondary modification of obligate horn growth regardless of male body size (Moczek and Nagy, 2005).

The same logic can of course be applied to sexual dimorphisms, that is, the absence of large horns in females of almost all species. Horns could have arisen as a malespecific trait right away from an ancestor in which both sexes were initially hornless. Alternatively, sexual horn dimorphisms could have arisen from an ancestor in which both sexes were initially horned, and female hornlessness evolved subsequently via a secondary loss through inhibition of horn growth in females only. As before, only in the latter case would we expect to see signs of horn growth or patterning, albeit possibly incomplete, in females. Interestingly, different hypotheses are supported for different horn types: male head horns, as in O. taurus, appear to develop in a strictly sex specific manner. Females never show signs of epidermal proliferation or patterning gene expression during prepupal development in this species, supporting the hypothesis that female hornlessness (for head horns) is most likely ancestral (Moczek and Nagy, 2005). In contrast, thoracic horns such as in O. nigriventris not only grow in females (but are then resorbed during the pupal stage, as explained earlier), but also express the same patterning genes during the prepupal growth phase as their male homologues. These results are consistent with the hypothesis that ancestrally both sexes may have expressed thoracic horns as adults, and that females lost their horn by evolving mechanisms to un-grow them, rather than not growing them in the first place. Combined, these data also suggest that thoracic and head horns may not only differ in the regulation of their initial growth, but also in subsequent mechanisms used to generate sexually dimorphic adults (Moczek, 2005). The notion that different types of horns may have evolved independently in the same clade has recently received further support by the first partial phylogenetic analysis of the genus Onthophagus (Emlen et al, 2005).

\section{The endocrine regulation of beetle horns}

The preceding section identified a variety of mechanisms that suggest that beetle horns and horn diversity are ultimately the product of a variety of processes, each likely to contribute its own degrees of freedom for subsequent diversification. One additional and critical set of processes controlling the development, but also the evolution of beetle horns, is their endocrine regulation, primarily via the common insect endocrine factors juvenile hormone $(\mathrm{JH})$, and possibly ecdysteroids.

$\mathrm{JH}$, is a sesquiterpenoid derived from farnesenic acid and is involved in almost every aspect of insect development and reproduction, including molting and metamorphosis, ovarian development and vitellogenin synthesis, caste determination in social insects and polyphenic development in locusts, aphids, and honey bees (Nijhout, 1994). Given its incredible versatility JH appeared as a prime candidate for the regulation of horn expression in beetles, and the differential regulation of horn expression in alternative male morphs or sexes. Studying the endocrine regulation of head horn development in O. taurus, Emlen and Nijhout (1999) found that treatment with the synthetic JH analog methoprene induced the expression of horns in males that normally would have developed into small hornless males, provided exposure to methoprene took place during a particular sensitive period late in larval development approximately $24-48 \mathrm{~h}$ prior to the gut purge. Based on this observation they suggested that male larvae may differ naturally in their $\mathrm{JH}$ titers depending on their body weight, and that there is a brief sensitive period during late larval development during which $\mathrm{JH}$ titers serve as a proxy for future adult body size. Under this model only male larvae heavy enough to express $\mathrm{JH}$ titers that exceed a certain titer threshold during this sensitive period will develop into horned males, whereas those exhibiting small body mass, and consequently, titers below the threshold, will become hornless. In a subsequent study, Emlen and Nijhout (2001) showed that methoprene treatment during an earlier developmental stage appears to have the opposite effect on male horn development. Now methoprene treated males developed relatively shorter horns (unfortunately measured on a qualitative scale form $0=$ no horns and $1=$ intermediate horns to $2=$ large horns rather than actual quantitative measurements) compared to untreated controls. Expanding their original model, the authors argued for a second, earlier sensitive period during which JH titers enable male larvae to decide between two alternative developmental pathways, horned or hornless, whereas JH titers experienced during the originally described, later sensitive period may regulate the exact amount of horn growth that occurs. Unfortunately, methoprene treatment during earlier developmental stages also highly significantly delayed the timing of pupation and the amount of time animals were therefore able to gain 
weight before the pupal molt. This raises the possibility that early methoprene treatment may actually alter body size of treated males while leaving horn development unaffected, generating males that for a given amount of horn growth develop relatively larger body sizes, rather than the other way around. Until methoprene effects on developmental timing can be dissociated from possible effects on growth and size the evidence in favor of a second earlier sensitive period for $\mathrm{JH}$, therefore, needs to be considered tentative.

Interestingly, Emlen and Nijhout (1999) also observed morph- and sex-specific differences in endocrine profiles for another common insect hormone, ecdysteroid. Ecdysteroids are sterol derivatives and the primary choreographer of the insect molting cycle (Nijhout, 1994). Emlen and Nijhout (1999) measured ecdysteroid titers in male and female larvae and prepupae and observed a rise of titers in all prepupae regardless of male morph or sex, which is expected and typical of animals that are nearing a molt. However, they also observed a small ecdysteroid peak during an earlier stage several days prior to this final rise of titers. This minor peak occurred only in female larvae and male larvae that would have developed into small hornless males, but not in larvae that would have given rise to large, horned males. This raised the possibility that this earlier ecdysteroid peak might somehow be involved in the induction of a female-like hornless morphology in small males, which would explain its presence in females and hornless males, but not in horned males. Lacking any functional data, however, this exciting hypothesis clearly requires further examination before given more weight. Furthermore, gene expression data published since (and partly discussed above) show unambiguously that horned and hornless male O. taurus, but not hornless males and females, share qualitatively similar patterning gene expression profiles. Although this does not necessarily rule out a role of ecdysteroids in horn expression it does contradict the notion that hornless morphologies in small males and females may arise through shared developmental control mechanisms.

Subsequent studies, however, confirmed the existence of the late $\mathrm{JH}$ sensitive period originally described by (Emlen and Nijhout, 1999; Moczek and Nijhout, 2002). More importantly, these studies also showed that evolutionary changes in several of its components provided important avenues for evolutionary changes in patterns of horn expression, ultimately driven by population-wide changes in ecological conditions (summarized in Figure 5; Moczek, 2003; Moczek and Nijhout, 2003). However, to fully appreciate how the endocrine regulation of beetle horns links ecological to evolutionary change, we first need to briefly review the behavioral and ecological context in which beetle horns and horned beetles function.

\section{Sexual selection, evolutionary endocrinology, and the diversification of horn allometries}

Regardless of the exact size, shape, or location of horn growth, all horned beetles studied thus far have been found to use their horns as weapons in male-male combat over access to mating opportunities (Eberhard, 1978; Siva-Jothy, 1987; Cook, 1990; Emlen, 1997; Eberhard et al, 2000; Moczek and Emlen, 2000; Hunt and Simmons, 2002; reviewed in Moczek (2005)). Males shove, prod, ram, lift, and throw their opponents to exclude others males, and long horns measurably increase male fighting success and fitness (Moczek and Emlen, 2000; Hunt and Simmons, 2001). Small, hornless males instead engage in nonaggressive sneaking tactics and rely largely on opportunistic satellite male behavior (Emlen, 1997). In species that compete within tunnels, such as most Onthophagus, behavioral assays suggest that the lack of horns is adaptive as it improves the sneaking performance of hornless males via enhancing maneuverability and agility inside the tunnel system in which small males search for mates (Emlen, 1997; Moczek and Emlen, 2000; Madewell and Moczek, in press). Alternative male morphs are therefore thought to be the product of disruptive selection acting on horn length, favoring long horns in males that fight, but hornlessness in males that engage in sneaking behavior. Under this scenario, intermediate morphologies are predicted to be both poor fighters and sneakers and thus be selected against in the context of either tactic. This mating system has several important consequences for the evolutionary ecology of horned beetles. First, males are predicted to switch from hornless to horned male morphologies at a body size at which the fitness gained from sneaking is just outweighed by the fitness gained from fighting. Second, the exact location of such a switch should at least in part depend on external conditions. Any ecological factor that somehow alters the composition of males within a population, or the nature of interactions between males, would be likely to also affect the exact location of the optimal body size threshold. Populations subject to divergent ecologies might then diverge in the exact scaling relationship between body size and horn length (Moczek, 2003).

Beetles in the genus Onthophagus provide an outstanding opportunity to examine if and how such divergences occur, and the ecological and developmental mechanisms that underlie them (Figure 5). O. taurus, the species that was already portrayed in detail with respect to the developmental genetic and endocrine regulation of horns, is also offering important insights into the evolutionary ecology of horned beetles. This species was originally limited to its native Mediterranean range, but in the early 1970s became introduced to western Australia as part of a biocontrol effort and accidentally to the eastern US around the same time. A combination of morphometric analyses of archival historic collections and quantitative genetic approaches showed that since introduction to their new habitat exotic O. taurus have diverged heritably in opposite directions relative to their Mediterranean ancestor and evolved highly divergent scaling relationships between body size and horn length (Moczek and Nijhout, 2003). While populations in each exotic range still produced both morphs, eastern US populations had shifted the body size threshold for horn induction to much smaller body sizes, causing even relatively small males to grow a full set of head horns. Western Australian populations, instead, had shifted their threshold size to much larger body sizes, causing only the very largest males to express horns. More importantly, threshold divergences between these populations were similar in kind and magnitude to many of the differences that we observe between true species, 

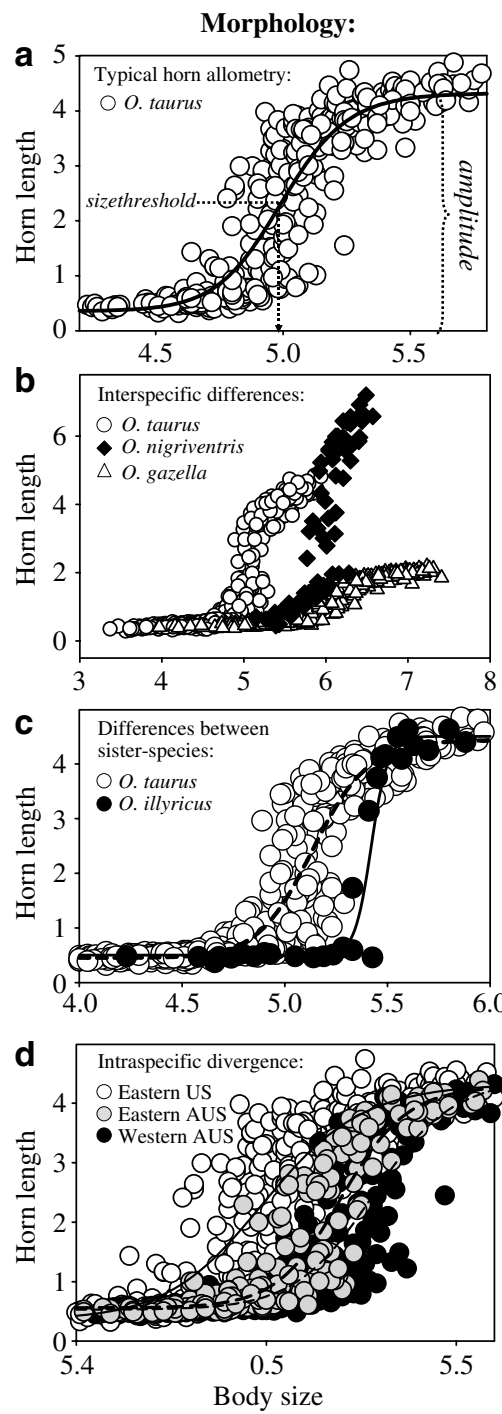

Behavioral ecology:

e

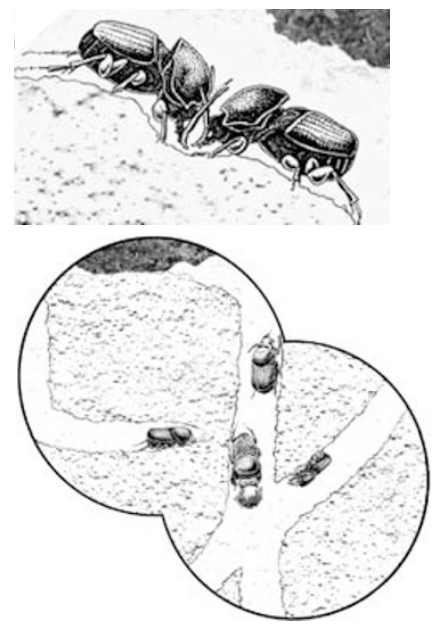

g

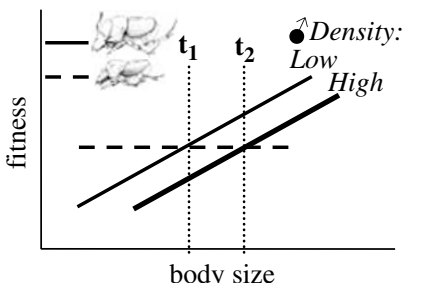

body size

h

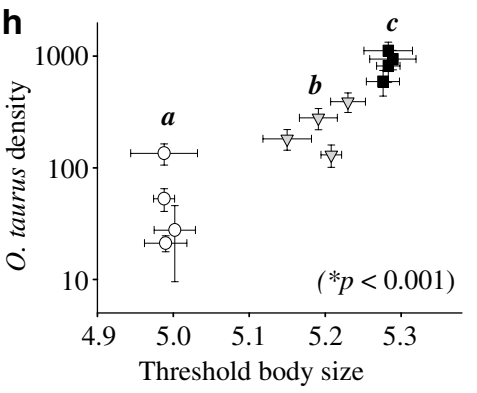

Development:

i

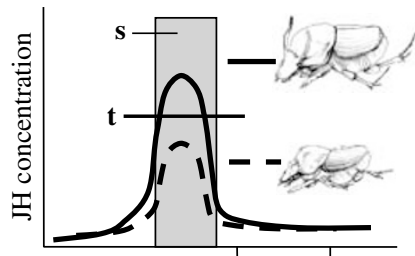

j

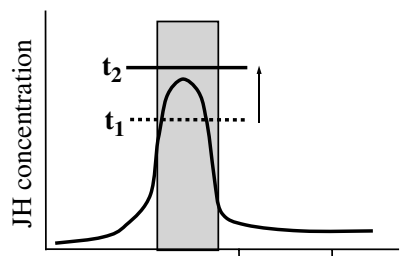

k

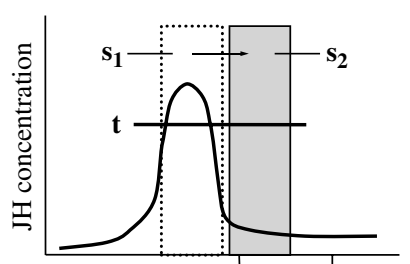

Figure 5 Integration of evolutionary morphology, behavioral ecology, and evolutionary endocrinology in the study of allometric diversity in Onthophagus beetles. Morphology: Evolutionary changes in allometric scaling provides important avenues for morphological diversification. (a) Scaling relationship (allometry) between body size ( $x$-axis) and horn length ( $y$-axis) of male O. taurus. The resulting sigmoid allometry is representative of numerous species of horned beetles in and outside the genus Onthophagus. (b) Comparisons to other Onthophagus species suggest that evolutionary changes in amplitude and threshold size (defined in (a)) have played an important part in the allometric diversification of Onthophagus beetles. (c) Comparisons between sister species suggest that threshold divergence may mark the first step in allometric diversification. (d) Allometric divergence between three rapidly diverging populations of $O$. taurus mirrors divergence patterns in the greater genus. Behavioral ecology: Changes in local ecologies drive allometric diversification. (e and $\mathbf{f}$ ) Alternative male morphs rely on alternative reproductive tactics to secure mating opportinuities. Horned males use thir horns as weapons in male-male combat. Hornless males rely on nonaggressive satellite-male behaviors to access females. (g) Males are predicted to switch from sneaking to fighting behavior at a threshold size $t$ at which the fitness gained from sneaking becomes outweighed by the fitness gained from fighting. The exact location of this intersection of fitness functions, and the corresponding optimal size thresholds for switching between alternative morphs, may change depending on external conditions. For example, increases in local densities of competing beetles are predicted to intensify male-male competition for females by increasing male encounter rates and decreasing the number of actively breeding females due to interference competition. As local densities increase, sneaking behavior becomes more profitable than fighting behavior over an increasing range of male body sizes, which in turn selects for a shift of the critical threshold body size $\boldsymbol{t}_{\mathbf{1}}$ to a larger body size $\boldsymbol{t}_{2}$. This hypothesis predicts a positive correlation between male threshold body sizes and local O. taurus densities, which is strongly supported by data collected from three natural, threshold-divergent populations (h). Development: Endocrine mechanisms of threshold evolution in onthophagine beetles. (i) Endocrine control of male horn dimorphism (modified after Emlen and Nijhout, 1999). Males are thought to differ in juvenile hormone (JH) titers as a function of their body size. Only males that exceed a certain size or weight express JH-titers above a threshold $(t)$ during a particular sensitive period (s), and in response will develop horns as adults. Smaller males with JH-titers below this threshold will remain hornless. (j) Elevation in the JH-threshold $\left(t_{1}\right.$ to $\left.t_{2}\right)$ prevents medium-sized male larvae to express JH-titers above the threshold necessary for horn development. Such larvae will now develop into the hornless instead of horned morph as adults. (k) A delay in the sensitive period for JH $\left(s_{1}\right.$ to $\left.s_{2}\right)$ causes the JH titer of medium-sized male larvae to fall below the JH-threshold necessary for horn induction before horn primordia become JHsensitive. As before, such males will now develop into the hornless instead of horned morph as adults ( $\mathrm{pp}=$ prepupal stage). (1) Experimental results from two threshold divergent populations (E-US and western Australia) support both hypotheses. Male larvae that normally would develop into the hornless morph were subjected to applications of the $\mathrm{JH}$ analogue Methoprene at different time points during late larval development ( $x$-axis). $Y$-axis indicated the percentage of males that responded to the treatment by switching developmental fate and developing horns. Horn induction in Australian males requires higher dosages (50 instead of $10 \mu \mathrm{g} / \mathrm{g}$ ) and exhibits delayed sensitivity compared to US males. Modified after Moczek and Nijhout (2002). 
which suggested that $O$. taurus populations may be undergoing evolutionary modifications similar to those that have helped past populations and species generate the allometric diversity present today (Figure 5a-d). These results also suggested that whatever caused these divergences, it could do so over extraordinarily short time periods.

Contrasting a large number of local populations in each of the two exotic ranges introduced above, as well as a third exotic range in eastern Australia, Moczek (2003) tested several hypotheses regarding the ecological causes of threshold divergences (Figure 5e-h). For example, threshold divergences might be brought about by population-wide changes in the distribution of male body sizes, causing the same absolute male size to be relatively large in one population, but relatively small in another. Alternatively, threshold divergences might be the product of changes in the intensity and nature of male-male competition, causing a particular tactic (eg fighting) to be advantageous over a wider range of body sizes in one population than another. Comparative analyses of these three allometrically divergent populations clearly rejected population-wide changes in the distribution of male body sizes as a possible explanation. However, major and consistent differences in local densities of competing males strongly supported the notion that rapid threshold divergences are driven by local differences in the intensity of male-male competition for females (Figure 5h).

Rapid threshold divergence between exotic O. taurus populations not only permitted major insights into the behavioral ecology of allometric divergence, but for the first time presented an opportunity to link these microevolutionary changes in horn expression to the underlying developmental mechanisms whose heritable modifications enabled such rapid evolution to take place (Figure 5i-1). The model of endocrine regulation of alternative male horn phenotypes originally put forward by Emlen and Nijhout (1999) suggested at least two major avenues of as to how the critical size threshold that separates horned and hornless males could become modified (Moczek and Nijhout, 2002). For example, by elevating the $\mathrm{JH}$ threshold required for horn induction, medium-sized males no longer produce a JH titer necessary for horn expression, and hence will develop into the hornless morph. Alternatively, a change in the timing of the sensitive period relative to $\mathrm{JH}$ secretion would also modify the critical threshold body size. For example, by delaying the sensitive period to just after the hormone peak, medium-sized males now again would express titers below the threshold, and would therefore develop into hornless males. On the level of a population both developmental modifications would generate a shift the body size threshold to larger body sizes. Comparative hormone manipulation experiments generated support for both hypotheses: horn induction in western Australian males is delayed subtly but significantly by $24-48 \mathrm{~h}$ and requires slightly, yet again significantly, higher hormone dosages compared to their eastern US counterparts (Figure 5h; Moczek and Nijhout, 2002). These results supported the hypothesis that relatively minor changes in the sensitivity to $\mathrm{JH}$ and its timing during larval development may be all it takes developmentally to allow beetle populations subject to simple, and probably ubiquitous differences in ecological conditions, to evolve novel allometries.

\section{Conclusions and outlook}

Beetle horns and horned beetles, in particular, of the genus Onthophagus, have recently become a successful model system for integrating the genetics, development, and ecology of morphological and developmental diversification. Representing a class of traits that lacks obvious homology to other insect structures, beetle horns are also providing increasing insight into the interplay between conservation and innovation in the origins of novel diversity. At the same time, we have barely begun to scratch the surface of what can yet be learned from these organisms. Approaches that are currently being developed include RNA interference protocols, genomic resources, and a further exploration of the interactions between endocrine and genetic regulators during horn development and diversification. With these new approaches, alongside already established tools, horned beetles are very likely to provide many more interesting, and probably surprising, insights into the genesis of diversity.

\section{Acknowledgements}

Tami Cruickshank, David VanDyken, Michele McDaniel, and Melanie O'Day provided helpful comments on earlier versions of this manuscript. Funding was provided in part through a National Institutes of Health Postdoctoral Excellence in Research and Teaching Fellowship (NIH Training Grant \# 1K12GM00708) and NSF Grant IOB 0445661 to the author.

\section{References}

Arrow GH (1951). Horned beetles. Junk Publishers: The Hague. Barolo S, Posakony JW (2002). The habits of highly effective signaling pathways: principles of transcriptional control by developmental cell signaling. Genes Dev 16: 1167-1181.

Campbell G (2002). Distalization of the Drosophila leg by graded EGF-receptor activity. Nature 418: 781-785.

Cook D (1990). Differences in courtship, mating and postcopulatory behavior between. male morphs of the dung beetle Onthophagus binodis Thunberg (Coleoptera: Scarabaeidae). Anim Behav 40: 428-436.

Eberhard WG (1978). Fighting behavior of male Golofa porteri beetles (Scarabaeidae: Dynastinae). Psyche 83: 292-298.

Eberhard WG, Garcia JM, Lobo J (2000). Size-specific defensive structures in a horned weevil confirm a classic battle plan: avoid fights with larger opponents. Proc R Soc London B 267: 1129-1134.

Emlen DJ (1997). Alternative reproductive tactics and maledimorphism in the horned beetle Onthophagus acuminatus (Coleoptera: Scarabaeidae). Behav Ecol Sociobiol 41: 335-341.

Emlen DJ, Allen CE (2004). Genotype to Phenotype: Physiological control of trait size and scaling in insects. Integrat Comparat Biol 43: 617-634

Emlen DJ, Marangelo J, Ball B, Cunningham CW (2005). Diversity in the weapons of sexual selection: horn evolution in the beetle genus Onthophagus (Coleoptera: Scarabaeidae). Evolution 59: 1060-1084.

Emlen DJ, Nijhout HF (1999). Hormonal control of male horn length dimorphism in the dung beetle Onthophagus taurus (Coleoptera: Scarabaeidae). J Insect Phys 45: 45-53.

Emlen DJ, Nijhout HF (2001). Hormonal Control of male horn length dimorphism in the dung beetle Onthophagus taurus 
(Coleoptera: Scarabaeidae): A second critical period of sensitivity to juvenile hormone. J Insect Phys 47: 1045-1054.

Fristrom D, Fristrom JW (1993). The metamorphic development of the adult epidermis. In: Bate M, Arias AM (eds) The development of Drosoplila melonogaster. Cold Spring Harbor Laboratory Press: New York. pp 843-897.

Galindo MI, Bishop SA, Greig S, Couso JP (2002). Leg patterning driven by proximal-distal interactions and EGFR signaling. Science 297: 256-259.

Hunt J, Simmons LW (2001). Status-dependent selection in the dimorphic beetle Onthophagus taurus. Proc Roy Soc London B 268: 2409-2414.

Hunt J, Simmons LW (2002). Confidence of paternity and paternal care: covariation revealed through experimental manipulation of a mating system in the beetle Onthophagus taurus. J Evol Biol 15: 784-795.

Kojima T (2004). The mechanism of Drosophila leg development along the proximodistal axis. Dev Growth Diff 46: 115-129.

Madewell R, Moczek AP (in press). Horn possession reduces maneuverability in a horn-polyphenic beetle. I Insert Sci.

Matthews EG (1972). A revision of the Scarabaeinae dung beetles of Australia. I. Tribe Onthophagini. Aus J Zool Suppl Ser 9: 1-330.

Miyawaki K, Inoue $\mathrm{Y}$, Mito T, Fujimoto T, Matsushima K, Shinmyo $\mathrm{Y}$ et al (2002). Expression patterns of aristaless in developing appendages of Gryllus bimaculatus (cricket). Mech Dev 113: 181-184.

Mizunuma T (1999). Giant Beetles. ESI Publishers: Tokyo, Japan.

Moczek AP (2003). The behavioral ecology of threshold evolution in a polyphenic beetle. Behav Ecol 14: 831-854.

Moczek AP (2005). Developmental plasticity and the origins of diversity: a case study on horned beetles. In: Ananthakrishnan TN, Whitman D (eds) Phenotypic Plasticity in Insects: Mechanisms and Consequences. Science Publishers, Inc: Plymouth, UK, in press.

Moczek AP (in press). Pupal remodeling and the development and evolution of sexual dimorphisms in horned beetles. Am Natural.

Moczek AP, Emlen DJ (1999). Proximate determination of male horn dimorphism in the beetle Onthophagus taurus (Coleoptera: Scarabaeidae). J Evol Biol 12: 27-37.
Moczek AP, Emlen DJ (2000). Male horn dimorphism in the scarab beetle Onthophagus taurus: do alternative reproductive tactics favor alternative phenotypes? Anim Behav 59: 459-466.

Moczek AP, Nagy LM (2005). Diverse developmental mechanisms contribute to different levels of diversity in horned beetles. Evol Dev 7: 175-185.

Moczek AP, Nijhout HF (2002). Developmental mechanisms of threshold evolution in a polyphenic beetle. Evol Dev 4: 252-264.

Moczek AP, Nijhout HF (2003). Rapid evolution of a polyphenic threshold. Evol Dev 5: 259-268.

Moczek AP, Rose D, Sewell W, Kesselring BR (in press). Conservation, innovation, and the evolution of horned beetle diversity. Dev Genes Evol.

Moczek AP, Cruickshank TE, Shelby JA (in review). When ontogeny reveals what phylogeny hides: gain and loss of horns during development and evolution of horned beetles. Evolution.

Nagy LM, Williams TA (2001). Comparative limb development as a tool for understanding the evolutionary diversification of limbs in arthropods: challenging the modularity paradigm. In: Wagner G (ed) The Character Concept in Evolutionary Biology. Academic Press: San Diego, CA, USA.

Nijhout HF (1991). The development and evolution of butterfly wing patterns. Smithsonian Institution Press: Washington, USA.

Nijhout HF (1994). Insect hormones. Princeton University Press: Princeton, USA.

Panganiban G, Irvine SM, Lowe C, Roehl H, Corley LS, Sherbon $B$ et al (1997). The origin and evolution of animal appendages. Proc Natl Acad Sci 94: 5162-5166.

Panganiban G, Nagy L, Carroll SB (1994). The role of the Distalless gene in the development and evolution of insect limbs. Curr Biol 4: 671-675.

Schneitz K, Spielmann P, Noll M (1993). Molecular genetics of aristaless, a prd-type homeobox gene involved in the morphogenesis of proximal and distal pattern elements in a subset of appendgaes in Drosophila. Genes Dev 16: 114-129.

Siva-Jothy MT (1987). Mate securing tactics and the cost of fighting in the Japanese horned beetle, Allomyrina dichotoma L. Scarabaeidae). J Ethol 5: 165-172.

Tomoyasu Y, Wheeler SR, Denell RE (2005). Ultrabithorax is required for membranous wing identity in the beetle Tribolium castaneum. Nature 433: 643-647. 